Trauma Berufskrankh 2008 10 [Suppl 1]:151-158

DOI 10.1007/s10039-007-1277-5

Online publiziert: 2. Dezember 2007

(c) Springer Medizin Verlag 2007

C. Luther · B. Bickert · G. Germann

Klinik für Hand, Plastische und Rekonstruktive Chirurgie -

Schwerbrandverletztenzentrum, BG-Unfallklinik Ludwigshafen, Klinik für

Plastische und Handchirurgie an der Universität Heidelberg, Ludwigshafen

\title{
Schmerzhaftes Handgelenk nach Sturz
}

gleicht. Er bildet zusammen mit dem M. pronator quadratus einen wichtigen Stabilisator des distalen Radioulnargelenks (DRUG).

Dorsal am Handgelenk verlaufen 12 Strecksehnen in 6 definierten Strecksehnenfächern, welche vom Retinaculum extensorum gebildet werden. Palmar am Handgelenk ziehen die 4 oberflächlichen und 4 tiefen Beugesehnen der Finger sowie die lange Daumenbeugesehne und der N. medianus durch den Karpalkanal, der N. ulnaris mit der A. ulnaris durch die Loge de Gyon zwischen Os pisiforme und dem Hamulus ossis hamati.

\section{Anamnese}

Die Unfallanamnese ist in 2 Fällen von Bedeutung:

1. Beim seltenen Hyperflexionstrauma des Handgelenks liegt oft eine Abscherfraktur des Tuberculum scaphoidei vor.

2. Ein Sturz aus einer Höhe schon ab $1 \mathrm{~m}$ führt häufig zu Bandverletzungen vom perilunären Typ.

Die derzeitigen Beschwerden, insbesondere die Schmerz auslösende Bewegung, sollten genau erfragt werden. So treten z. B. bei radialseitigen Bandinstabilitäten eher Schmerzen, bei ulnarseitigen eher Schnappphänomene auf.

Akute, subakute und chronische Handgelenkschmerzen [7]. Von einer akuten Handgelenkverletzung spricht man bis zur 2. Woche nach dem Trauma, von einer subakuten Verletzung zwischen der 2. und 6. Woche und von einer chronischen Symptomatik, wenn die Beschwerden länger als 6 Wochen andauern.

\section{Diagnostische Verfahren}

\section{Röntgen}

Nach einem Sturz auf die Hand müssen zunächst knöcherne Verletzungen durch Nativröntgenaufnahmen des Handgelenks abgeklärt werden, die die gesamte Handwurzel in 4 Ebenen beinhalten sollten:

- posterior-anterior,

- seitlich in exakter Neutralstellung,

- Handgelenk $20^{\circ}$ proniert (Moneim) und

- $20^{\circ}$ supiniert in leichter Ulnadeviation (Stecher).

Eine Röntgenaufnahme des Handgelenkes nach Moneim kann auf eine Verletzung des skapholunären Bands (Vergrößerung des skapholunären Winkels) Hinweis geben (- Abb. 2, 3). Bei einer Röntgenaufnahme nach Stecher ist das Skaphoid in voller Längsachse dargestellt, sodass eine Skaphoidfraktur am besten sichtbar wird (• Abb. 4) [12]. Bei Sturz aus der Höhe sollte der gesamte Unterarm mit Ellenbogen in 2 Ebenen geröntgt werden, um eine Essex-Lopresti-Verletzung auszuschließen (s. unten).

Bei normalem Röntgenbefund sollte das Handgelenk unter abschwellenden Maßnahmen einige Tage ruhig gestellt und anschließend, bei abgeklungener Schmerzsymptomatik, erneut untersucht werden. 


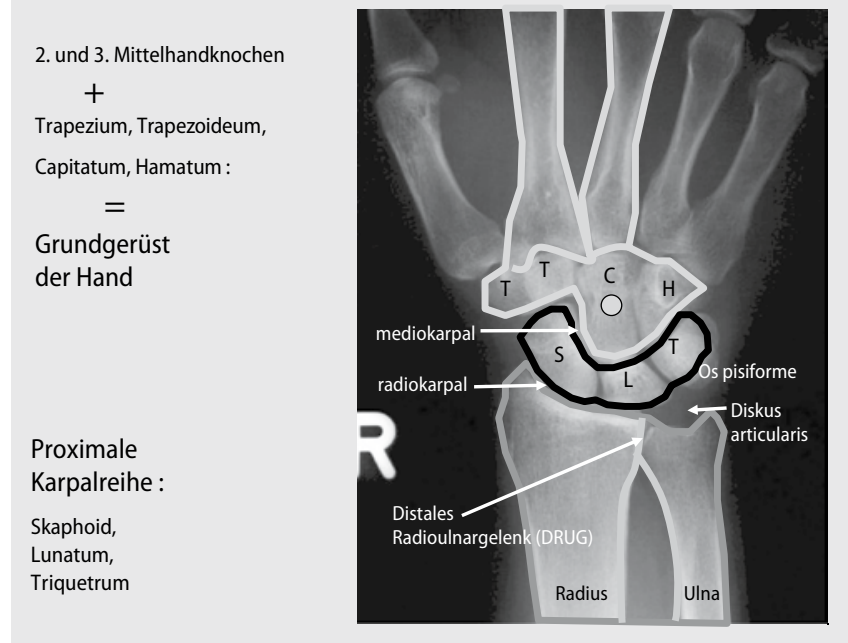

Abb. $1 \varangle$ Anatomie des Handgelenks und der Hand
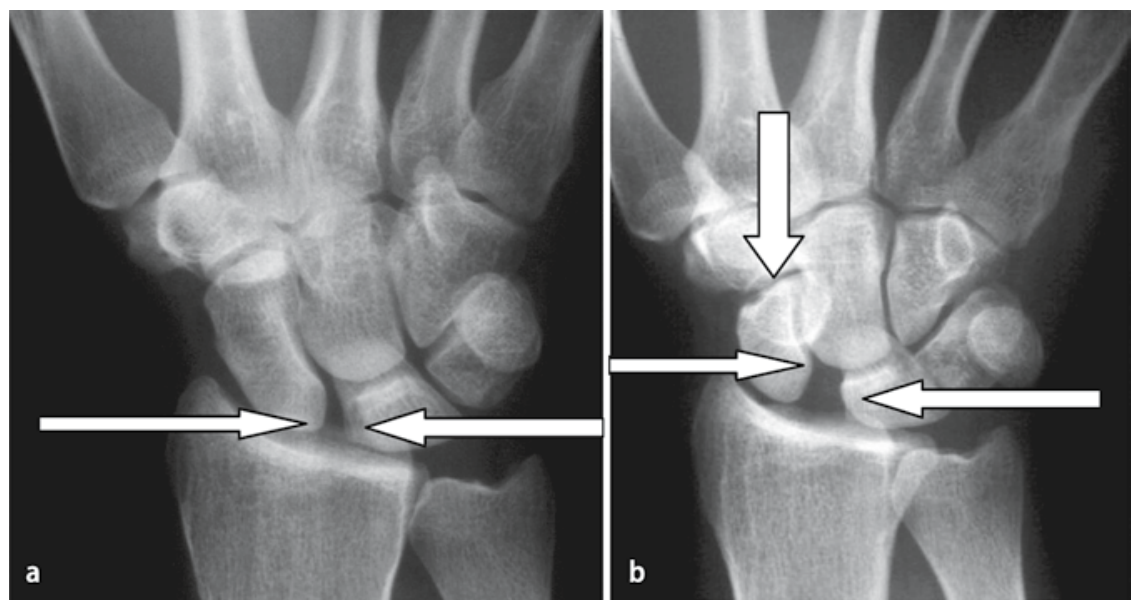

Abb. 2 \ Skapholunäre Bandruptur, a im p.-a.Röntgenbild erweiterter SL-Spalt sichtbar, entspricht SL-Dissoziation (Pfeile), b im Röntgenbild nach Moneim erweiterter SL-Spalt (dünne Pfeile), Skaphoid nach palmar verkippt (Ringzeichen des Skaphoids, dicker Pfeil)
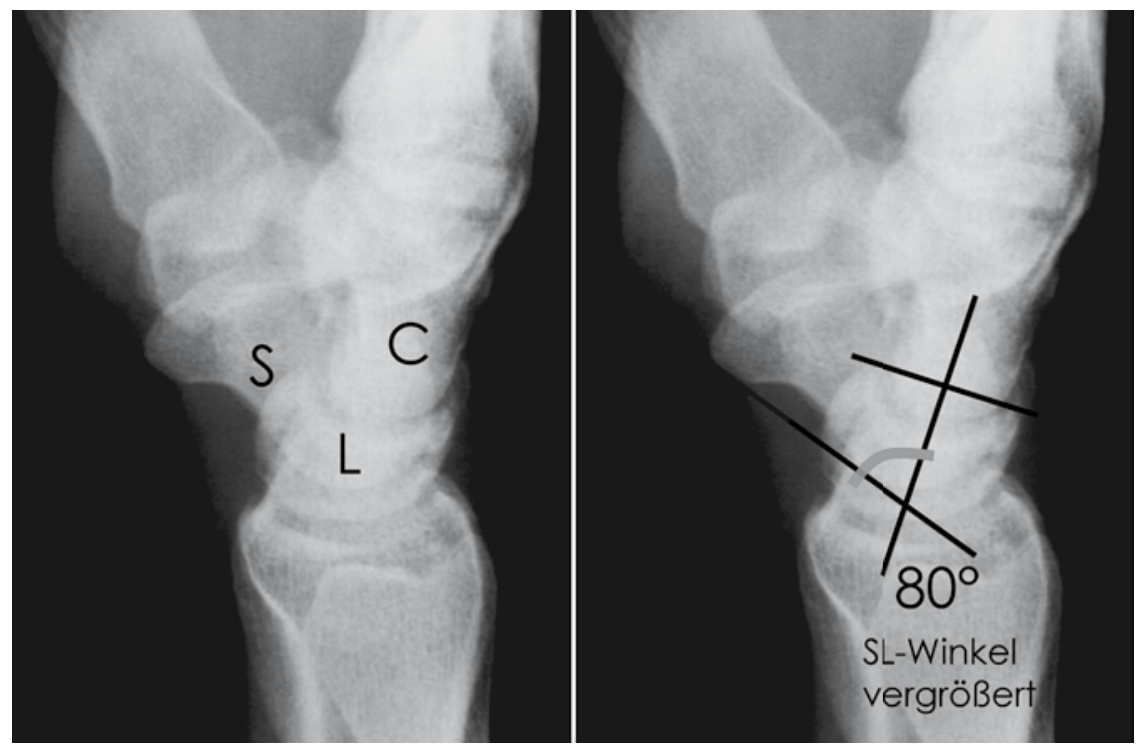

Abb. 3 \ Skapholunäre Dissoziation, im seitlichen Röntgenbild SL-Winkel vergrößert $\left(80^{\circ}\right)$

\section{Computertomographie}

Sie gibt die zuverlässigste Aussage über eine knöcherne Beteiligung der Handwurzel oder Dislokation einer Fraktur [3]. Bei Schmerzpersistenz, v. a. bei Druckschmerz in der Tabatiere, sollte eine Computertomographie der Handwurzel erfolgen, um eine Skaphoidfraktur oder andere begleitende Handwurzelfrakturen sicher auszuschließen. Hierbei sollte das CT immer in Längsachse des Skaphoids mit 1-mm-Schichtung durchgeführt werden (• Abb. 5).

\section{Magnetresonanztomographie}

Sie kann zur Beurteilung ligamentärer Verletzungen eingesetzt werden, insbesondere des TFCC [18]. Bei chronischem Handgelenkschmerz sind unter zusätzlicher Gabe des Kontrastmittels Gadolinium eine avaskuläre Knochennekrose des Lunatums (Morbus Kienböck), des Skaphoids (Morbus Preiser) oder ein schmerzhaftes intraossäres Ganglion zuverlässig nachweisbar.

\section{Kinematographie}

Eine Instabilität der Handwurzel, z. B. zwischen Skaphoid und Lunatum bei Ruptur des skapholunären Bandes oder im DRUG, lässt sich durch eine dynamische Durchleuchtung (Kinematographie) nachweisen.

\section{Handgelenkarthroskopie}

Sie sollte bei klinisch/radiologischem Verdacht auf eine intraartikuläre Pathologie des Handgelenks durchgeführt werden. Hierbei können v. a. Knorpelschäden an den Gelenkflächen im Radio- (zwischen Radius/Ulna und proximaler Handwurzelreihe) und Mediokarpalgelenk (zwischen proximaler und distaler Handwurzelreihe), aber auch eine Ruptur des TFCC oder eine posttraumatische dynamische Instabilität (SL- oder LT-Instabilität) sicher beurteilt werden (• Abb. 6). Bei zentralen Diskusrissen erfolgt ein Diskusdébridement, um schmerzhafte Einklemmungen des Diskus bei Bewegung zu vermeiden. Bei Bandläsionen (SL- und LT-Band) muss beurteilt werden, ob eine 


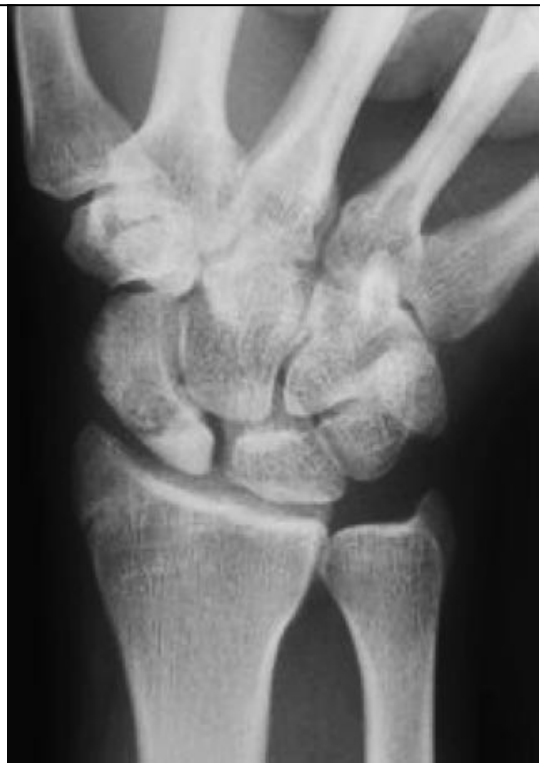

Abb. $4 \Delta$ Stecher-Aufnahme des Handgelenks, Skaphoidfraktur, proximaler Pol

Bandrekonstruktion sekundär möglich ist (Kontraindikation bei bereits vorhandenen Knorpelschäden der Gelenkflächen).

\section{MR-Arthrographie}

Die Kombination von Arthrographie (Injektion von Kontrastmittel ins Handgelenk) und Magnetresonanztomographie kann möglicherweise die Anzahl rein diagnostischer Handgelenkarthroskopien (ohne pathologischen Befund) reduzieren $[8]$.

\section{Klinische Untersuchung}

Einen Überblick gibt $\bullet$ Tab. $1[1,4,10]$.

\section{Inspektion des Handgelenks}

Der Patient sollte die betroffene und die unverletzte Hand locker auf dem Untersuchungstisch lagern. Es sollte geachtet werden auf:

- lokalisierte Schwellungen (als Hinweis auf eine Synovialitis),

- trophische Störungen der Haut [z. B. beim CRPS (komplexes regionales Schmerzsyndrom)],

- Fehlstellungen: z. B. Bajonettstellung nach fehlverheilter distaler $\mathrm{Ra}$ diusfraktur oder die von ulnar zu sehende palmarwärtige Verschiebung der Handwurzel bei mediokarpaler Handwurzelinstabilität,

Trauma Berufskrankh 2008 · 10[Suppl 1]:151-158 DOI 10.1007/s10039-007-1277-5

(c) Springer Medizin Verlag 2007

\section{Luther · B. Bickert · G. Germann}

\section{Schmerzhaftes Handgelenk nach Sturz}

\section{Zusammenfassung}

Die zugrunde liegende Diagnose eines traumatisch bedingten Handgelenkschmerzes kann durch Anamnese, Inspektion, Palpation der Landmarke des Handgelenks und spezifische Provokationsstests zur Überprüfung von Bandstabilitäten sehr präzise erfasst werden. Die gängigen Untersuchungsverfahren sind v. a. für den in der Praxis tätigen Chirurgen leicht erlernbar und von großer klinischer Relevanz, da die häufig erforderliche Zuweisung in spezialisierte Handzentren von der Einschätzung des primär behandelnden Arztes abhängt. Neben den Nativröntgenaufnahmen können Zusatzuntersuchungen wie Computertomographie, Magnetresonanztomographie oder Kinematographie des Handgelenks wertvolle Informationen geben. Die Handgelenkarthroskopie ist noch immer un-

\section{Wrist-pain following a fall}

\section{Abstract}

The diagnosis underlying posttraumatic wrist pain can be very precisely determined from the patient's history, examination, palpation of the landmarks of the wrist and specific provocation tests to test for ligamentous stability. The routine investigation methods for the wrist are easy to learn, especially for practising surgeons, and very relevant to the clinical course, since it is frequently necessary for patients to be referred to specialised hand clinics and the first doctor to see the patient is responsible for deciding about this. Besides plain X-rays of the wrist, further diagnostic investigations, such as computed tomography, magnetic resonance imaging or verzichtbar in der Beurteilung intraartikulärer Pathologien wie Bandrupturen, Läsionen des triangulären fibrokartilaginären Komplexes ("triangular fibrocartilge complex", TFCC) oder Knorpelschäden im Radio- und Mediokarpalgelenk. Mit dieser Übersichtsarbeit möchten wir einen Einblick geben in die klinischen Untersuchungstechniken, diagnostischen Verfahren und Differenzialdiagnosen des traumatisch bedingten Handgelenkschmerzes.

\section{Schlüsselwörter}

Verletzungen des Handgelenks · Triangulärer fibrokartilaginärer Komplex (TFCC) .

Magnetresonanztomographie · Handgelenkarthroskopie · Spezialisiertes Handzentrum cinematograpy are helpful. Arthroscopy of the wrist is still essential in the assessment of such intraarticular pathologies as ligament ruptures, lesions of the triangular fibrocartilage complex (TFCC) or cartilaginous lesions in the radiocarpal or midcarpal joint. We hope with this review to give a comprehensive insight into the clinical investigation techniquess, diagnostic procedures and differential diagnosis of posttraumatic wrist pain.

\section{Keywords}

Wrist injuries - Triangular fibrocartilage complex - Magnetic resonance imaging · Arthroscopy of the wrist $\cdot$ Specialised wrist unit 


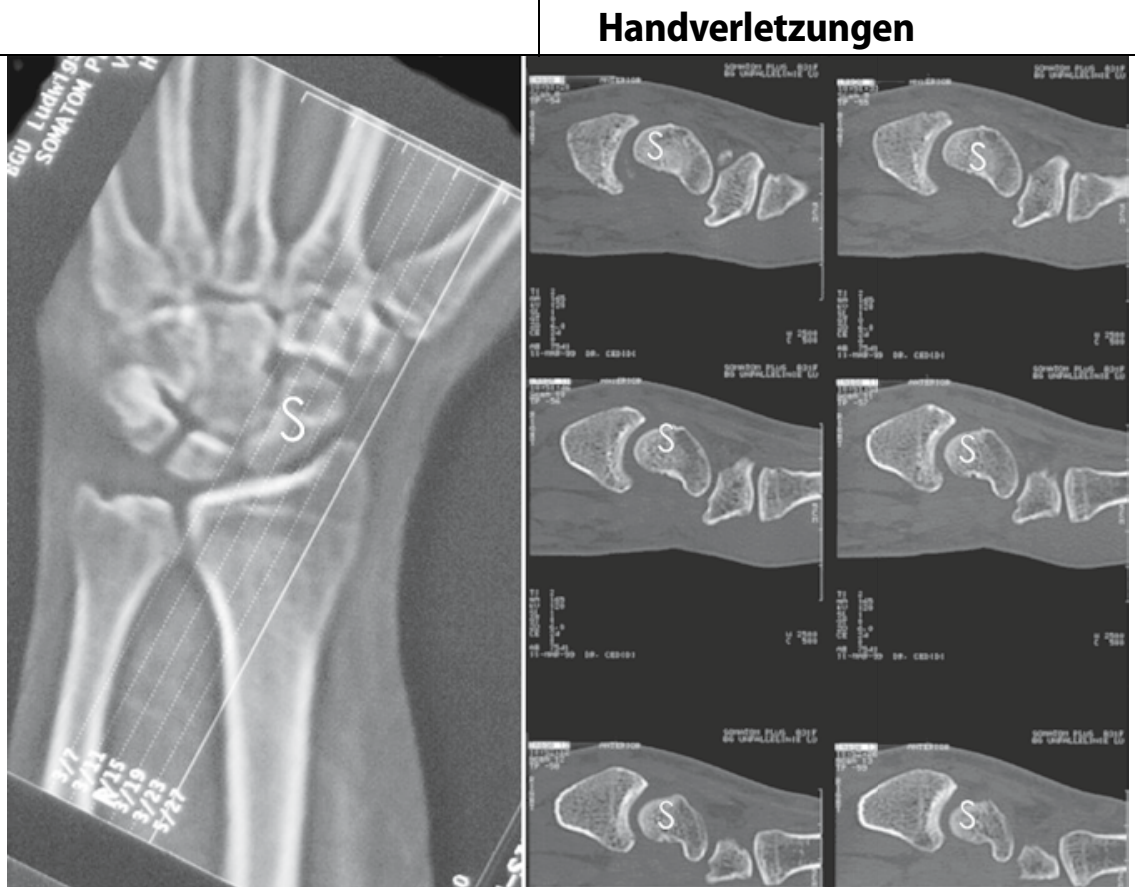

Abb. 5 ム CT der Handwurzel (in Skaphoidlängsachse und 1-mm-Schichten)

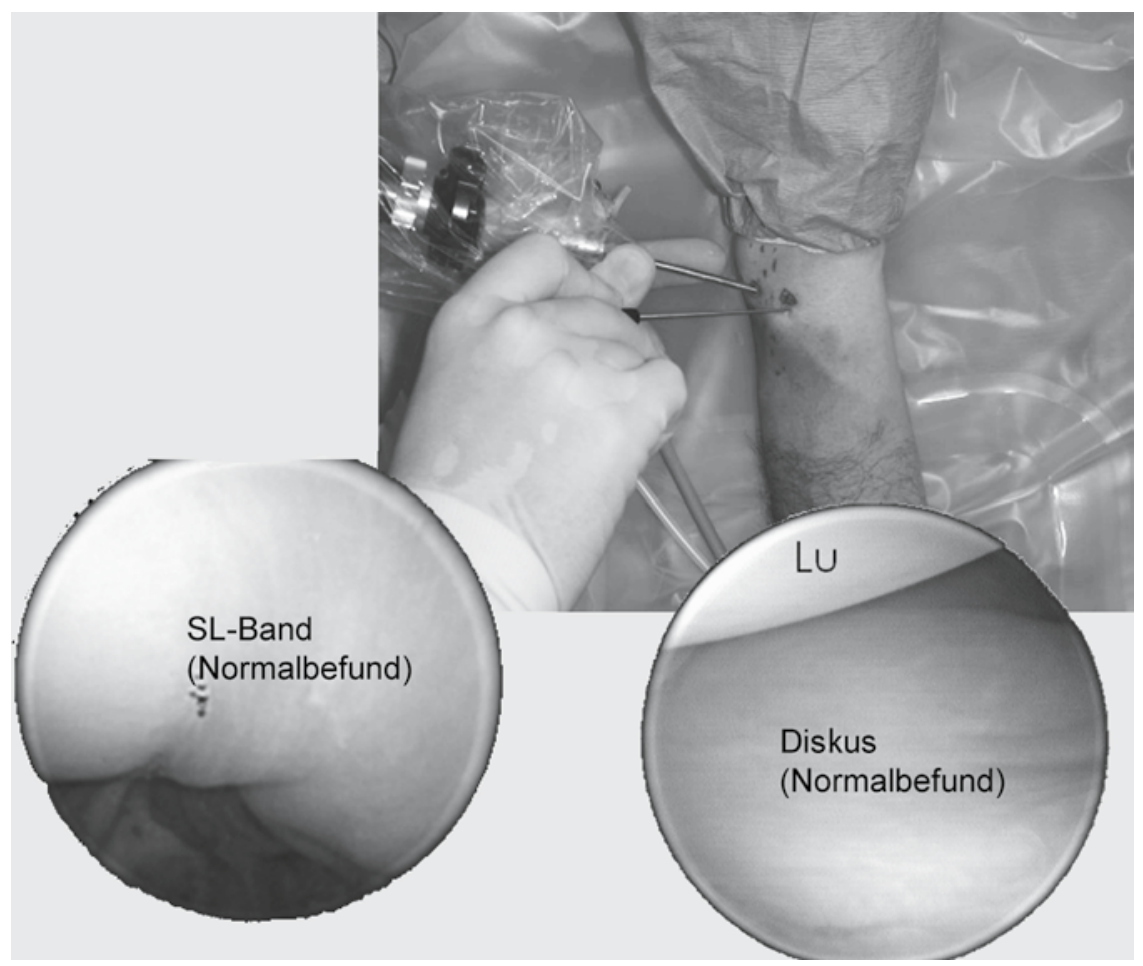

Abb. $6 \Delta$ Handgelenkarthroskopie

- Konturen beider Handgelenke im Vergleich: das in Pronation prominente Ulnaköpfchen könnte Ausdruck einer möglichen Instabilität des distalen Radioulnargelenks (DRUG) sein,

- Atrophien der Muskulatur: z. B. Thenaratrophie beim posttraumatischen Karpaltunnelsyndrom oder
Atrophie der Mm. interossei als Hinweis auf eine N.-ulnaris-Lähmung.

\section{Palpation des Handgelenks}

Schmerzhafte Druckpunkte am Handgelenk sollten exakt lokalisiert werden, wobei auf folgende Pathologien zu achten ist (- Abb. 7):
- in der Tabatiere und Stauchungsschmerz des Daumens

- über dem Processus styloideus radii (Griffelfortsatz der Speiche)

- über dem skapholunären Band (SLBand): $1 \mathrm{~cm}$ distal des Tuberculum Listeri am radialen Rand des 4. Strecksehnenfachs tastbar

- über dem lunotriquetralen Band (LT-Band): $1 \mathrm{~cm}$ distal des Tuberculum Listeri, am ulnaren Rand des 4. Strecksehnenfachs tastbar

- über dem STT-Gelenk (Gelenk zwischen Skaphoid, Trapezium und Trapezoideum): unmittelbar distal der Spitze des Processus styloideus radii

- über dem Daumensattelgelenk (zwischen Trapezium und Basis des 1. Mittelhandknochens)

- über dem Os pisiforme (Knochenvorsprung am beugeseitigen Handgelenk lateral) über der Fovea: am distalen Rand des Ulnaköpfchens zwischen der Sehne des M. flexor und des M. extensor carpi ulnaris (• Abb. 8) [16]

- über den Karpometakarpalgelenken (CMC 2-5)

\section{Provokationstests am Handgelenk}

Hierbei werden dynamische Instabilitäten durch Provokation einer Subluxation aufgedeckt. Die Tests sollten immer im Seitenvergleich durchgeführt werden, da bei Laxizität des Bandapparats seitengleiche Instabilitäten oft keine klinische Relevanz haben. Jede Instabilität sollte als statisch (schon durch Inspektion oder auf Nativröntgenaufnahmen zu erkennen) oder dynamisch (nur durch Provokation auslösbar) klassifiziert werden. Die statischen Instabilitäten sollten klinisch auf ihre Reponierbarkeit („statisch-reponierbar" oder „statisch-irreponierbar") getestet werden [6].

Skaphoid-Shift-Test nach Watson [17]. Er dient der Diagnose einer skapholunären Bandverletzung oder einer Rotationsinstabilität des Skaphoids. Bei Radialduktion der Hand wird das Skaphoid durch Druck des Trapeziums nach palmar flektiert, bei Ulnarduktion in eine relative Streckstellung zur Radiuslängsachse gezogen. 
Die rechte Hand des Untersuchers greift von radial das rechte Handgelenk des Patienten (bzw. links auf links), sodass der Daumen Druck aufs Tuberculum scaphoidei ausübt und Zeige- und Mittelfinger dorsal das SL-Gelenk tasten können. Wird nun die Hand des Patienten von ulnar nach radial geführt, drückt das Skaphoid den Daumen des Untersuchers nach palmar. Der Test ist positiv und somit pathologisch, wenn der Daumendruck das Skaphoid nach dorsal subluxieren lässt mit Schmerzen im skapholunären Gelenk (• Abb. 9).

Skapholunärer Ballottementtest. Im Anschluss an den Watson-Test wird das Skaphoid weiter zwischen Daumen und Zeigefinger gehalten. Die andere Hand fixiert das Lunatum. Dann wird das Skaphoid in rascher Folge in dorso-palmarer Richtung rasch hin- und herballottiert, was bei SL-Instabilität schmerzhaft ist.

\section{Lunatotriquetraler Ballottementtest.} Ähnlich dem SL-Ballottementtest wird das Lunatum gegen das Triquetrum in dorsopalmarer Richtung rasch hin- und herballotiert, was bei LT-Instabilität schmerzhaft ist.

Diskus-Grinding-Test. Es wird eine axiale Kompression der Hand in Ulnardeviation mit dorso-palmarer Schiebebewegung, abwechselnd in Pro- und Supination, durchgeführt. Dazu fixiert der Untersucher mit einer Hand den Unterarm des Patienten. Die andere Hand rotiert die ulnarduzierte Hand des Patienten. Ist dies schmerzhaft, ist der Test positiv. Es kann sich um eine Diskusläsion, eine lokale Synovialreaktion oder einen Knorpelschaden im LT-Bereich handeln ( $\bullet$ Abb. 10).

Klaviertastentest. Ein in dorsaler Subluxation stehendes Ulnaköpfchen wird in Pronation nach palmar reponiert, was schmerzhaft sein kann. Beim Loslassen gleitet das Ulnaköpfchen in die subluxierte Position zurück und deutet auf eine dorsale Bandruptur des DRUG hin.

DRUG-Shift-Test. Der Ulnakopf wird gegen den distalen Radius mit Kraft dorso-palmar hin und her bewegt. Ist di-
Abb. 7 Klinische Untersuchung des Handgelenks: Palpation der Landmarken

Abb. 8 Foveazeichen: Druckschmerz zwischen Flexor-carpi-ulnaris- und Extensor-carpi-ulnarisSehne distal des UInakopfs

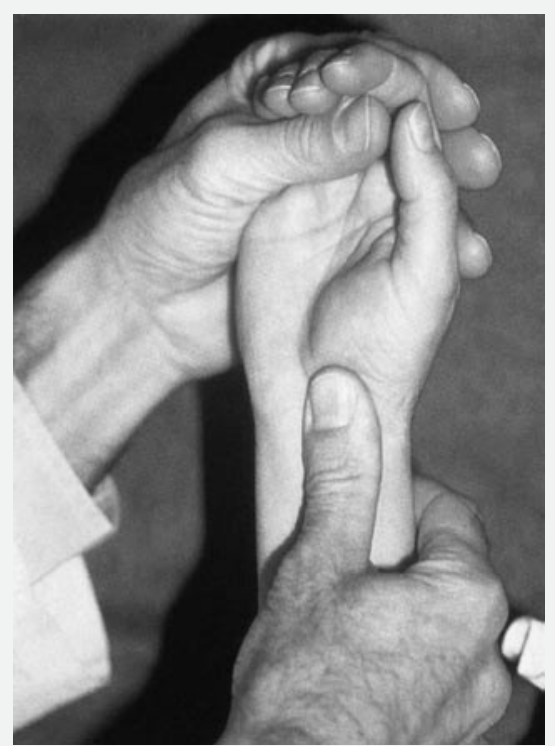

Abb. $9 \Delta$ Skaphoid-Shift-Test nach Watson

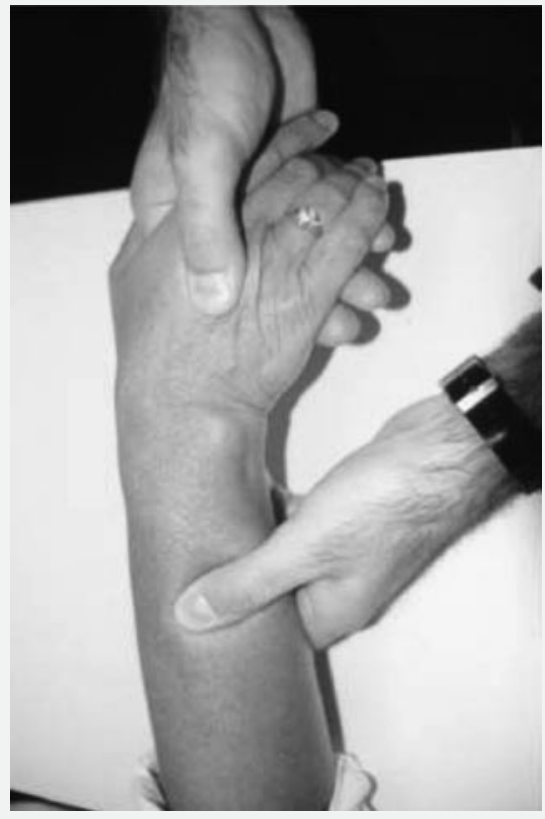

Abb. $10 \Delta$ Ulnarer Stresstest, ulnares Grinding 


\begin{tabular}{|ll|}
\hline $\begin{array}{l}\text { Tab. } 1 \text { Klinische Untersuchung des } \\
\text { Handgelenks }\end{array}$ & $\begin{array}{l}\text { Schwellung } \\
\text { Trophik/Atrophie } \\
\text { Asymmetrie } \\
\text { Fehlstellung }\end{array}$ \\
& $\begin{array}{l}\text { Landmarken } \\
\text { Provokationstests }\end{array}$ \\
\hline Palpation & $\begin{array}{l}\text { Aktive und passive } \\
\text { Beweglichkeit } \\
\text { Kraft }\end{array}$ \\
\hline Motorik & \\
\hline Sensibilität & \\
\hline
\end{tabular}

es schmerzhaft, kann eine Arthrose im DRUG vorliegen.

Catch-up-Clunk-Test. Der Patient führt die geballte Faust von radial nach ulnar hin und her. Bei positivem Test ist ein oft schmerzhaftes Schnappen spürbar, was auf eine Instabilität im Mediokarpalgelenk hindeutet.

Finkelstein-Test. Die Ulnardeviation führt zu mäßigen, bei in der Faust eingeschlagenem Daumen heftigen Schmerzen im 1. Strecksehnenfach, was auf eine Tendovaginitis de Quervain hinweist (schmerzhafte Einengung der Sehne des M. abductor pollicis longus und extensor pollicis brevis).

Dorso-palmare Translation der Handwurzel bei fixiertem Unterarm. Die Hand wird in Höhe des Capitatums fixiert und nach dorso-palmar hin- und hergeschoben. Eine dorsale Translation deutet auf eine Ruptur des distalen V-Bands hin.

\section{Untersuchung bei Sensibili- tätsstörungen der Hand}

Hinweisend auf ein Karpaltunnelsyndrom sind:

- Hofmann-Tinell-Zeichen: elektrisierende Missempfindungen in Daumen, Zeige- und Mittelfinger bei Beklopfen des Karpalkanals

- Phalen-Test: Aneinanderlegen der Handrücken bei $90^{\circ}$ Beugung im Handgelenk löst bei positivem Test Kribbelmissempfindungen in Daumen, Zeige- und Mittelfinger aus
- Durkan-Zeichen: Positiv bei Kribbelmissempfindungen in Daumen, Zeige- und Mittelfinger bei Kompression des Karpaltunnels

\section{Klinische Differenzialdiagnosen des schmerzhaften Handgelenks nach Sturz}

Distale Radiusfraktur. Sie kann mit einer Skaphoidfraktur oder SL-Band-Läsion kombiniert sein, v. a. bei intraartikulärem Frakturverlauf. Zum Ausschluss einer Skaphoidfraktur sollte präoperativ eine Computertomographie durchgeführt werden. Ist eine Osteosynthese der Radiusfraktur erforderlich, kann die intraoperative Durchbewegung des Handgelenks unter dem Bildwandler nach Osteosynthese des Radius Hinweis auf eine SLBand-Läsion geben, wenn der SL-Spalt bei Radial- bzw. Ulnaduktion klafft. Ist keine Operation der Radiusfraktur notwendig, kann eine sekundär durchgeführte Handgelenkspiegelung den klinisch/radiologischen Verdacht auf eine SL-Dissoziation abklären.

Instabilität und/oder Arthrose des distalen Radioulnargelenks (DRUG). Durch eine schwere Distorsion des Handgelenks, v. a. in Pronation und Dorsalextension, kann es zur Zerreißung der radioulnaren Bänder kommen mit federnder, leicht prominenter Dorsalverschiebung des Ulnakopfs und Subluxationsneigung. Bei der klinischen Untersuchung zeigt sich eine oft schmerzhafte, krepitierende dorsovolare Verschieblichkeit des Ulnaköpfchens (DRUG-Shift-Test). Auf der Röntgen-p.a.-Aufnahme des Handgelenks kann eine distale radioulnare Diastase als Ausdruck der Diskuszerreißung vorhanden sein; auf der exakt seitlichen Röntgenaufnahme ist eine Dorsalverschiebung des Ellenkopfs erkennbar $[5,9]$.

Diskusriss bzw. Läsion des TFCC. Es besteht ein ulnokarpaler Handgelenkschmerz, der gelegentlich mit Schnappphänomenen verbunden ist. Fovea- und Ulna-Grinding-Test sind positiv.

SL-Band-Ruptur. Typische Zeichen sind radiokarpale Schmerzen, Druckschmerz über dem SL-Band, positiver Watson- und positiver skapholunärer Ballottementtest (• Abb. 11).

Moneim- (dynamische Instabilität) bzw. p.-a-Röntgenaufnahme (statische Instabilität) zeigen einen erweiterten SLSpalt, im seitlichen Röntgenbild ist das Lunatum nach dorsal verkippt (DISI-Stellung, Abb. 3). Es wird eine Handgelenkarthroskopie empfohlen.

LT-Band-Ruptur. Es bestehen Schmerzen und/oder Schnappen ulnokarpal sowie ein Druckschmerz über dem LT-Band. Der lunatotriquetrale Ballottementtest ist positiv. In der p.-a.-Röntgenaufnahme ist evtl. ein erweiterter LT-Spalt sichtbar, im seitlichen Röntgenbild erscheint das Lunatum nach palmar verkippt (PISI-Stellung). Es wird eine Handgelenkarthroskopie empfohlen.

Skaphoidfraktur. Typisch sind ein Druckschmerz in der Tabatiere sowie ein Stauchungsschmerz des Daumens. Zur exakten Diagnose sind Röntgenaufnahme in 4 Ebenen, v. a. eine Stecher-Aufnahme, notwendig. Zudem ist ein CT indiziert, um eine Abkippung der Fraktur und die eventuelle Operationspflichtigkeit zu überprüfen (• Abb. 12, 13) [3, 12].

Handgelenkarthrose nach veralteter Skaphoidfraktur (SNAC-Wrist). Als Folge einer unbehandelten Skaphoidfraktur kollabiert der Carpus und verursacht Schmerzen im Handgelenk („scaphoid non-union advanced collapse“, SNACWrist) (• Abb. 14).

Handgelenkarthrose nach veralteter SL-Band-Läsion (SLAC-Wrist). Als Folge einer unbehandelten skapholunären Bandruptur kollabiert der Carpus und verursacht Schmerzen im Handgelenk („scapho-lunate advanced collapse“, SLAC-Wrist). Bei SNAC- und SLAC-wrist im frühen Stadium ist noch eine rekonstruktive Operation an Skaphoid bzw. SLBand möglich, im fortgeschrittenen Stadium werden ggf. eine Teilversteifung und/ oder Denervierung des Handgelenks erforderlich [13].

Aktivierte Arthrose. Sie äußert sich in Schmerzen und Schwellung des gesamt- 


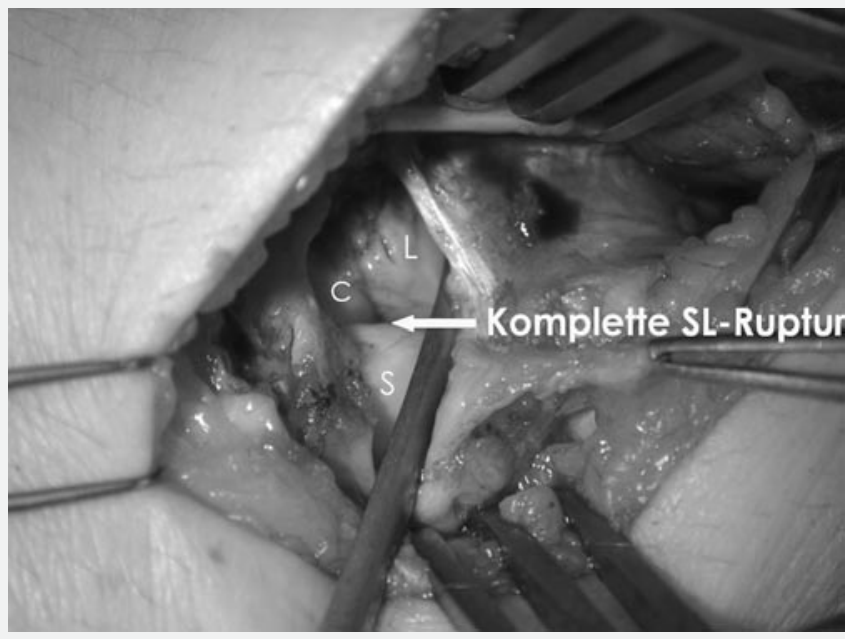

Abb. 11 A Intraoperativer Situs bei kompletter skapholunärer Bandruptur

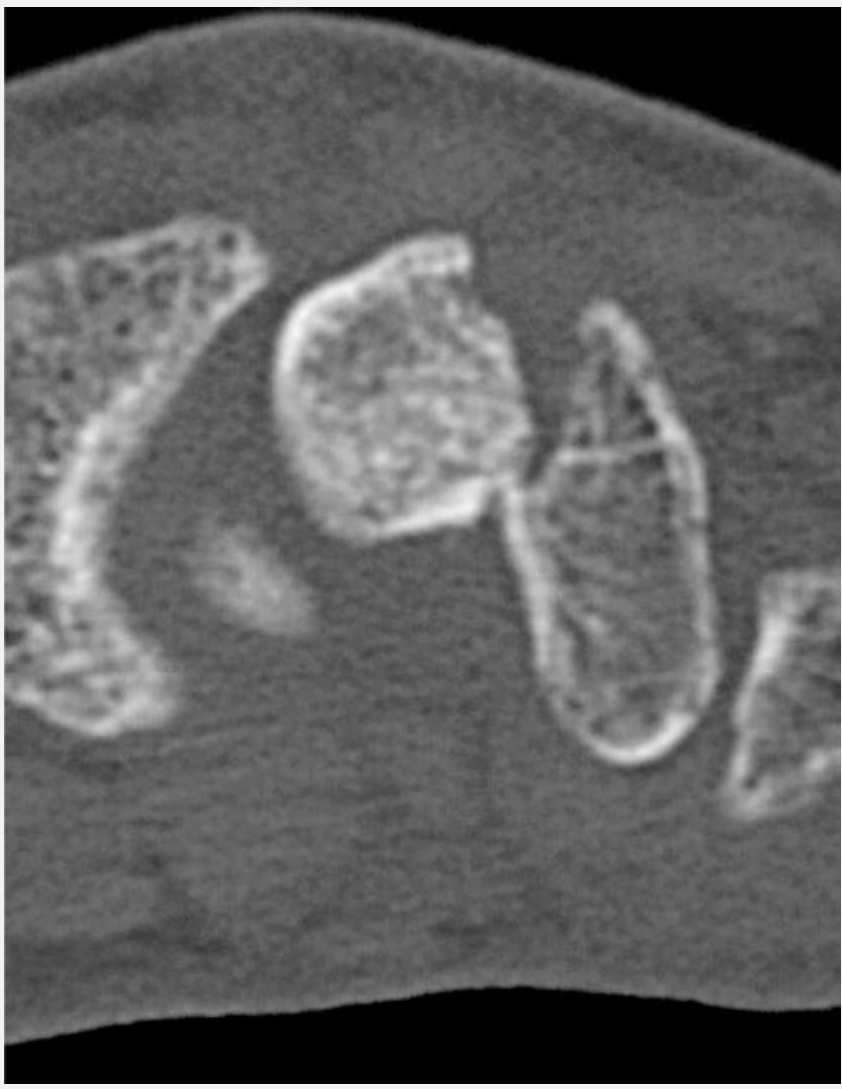

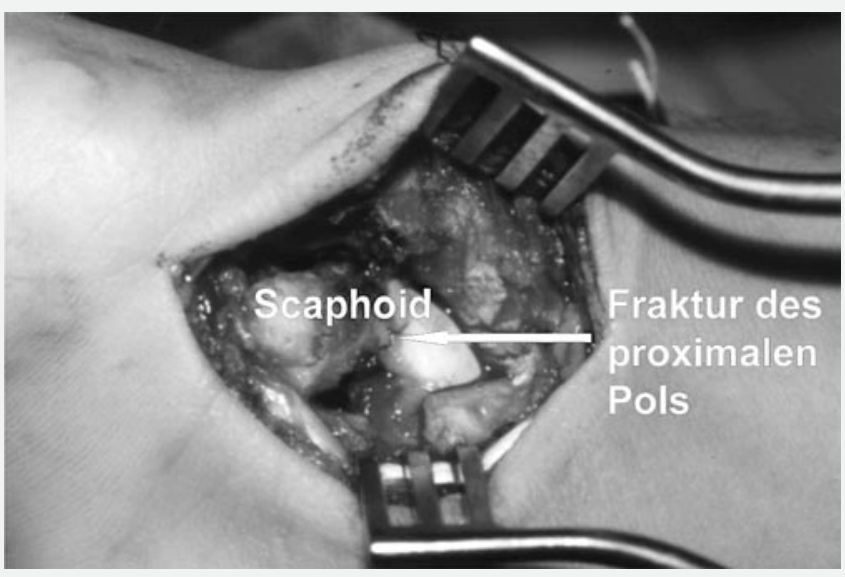

Abb. $12 \Delta$ Intraoperativer Situs bei Fraktur des proximalen Skaphoidpols

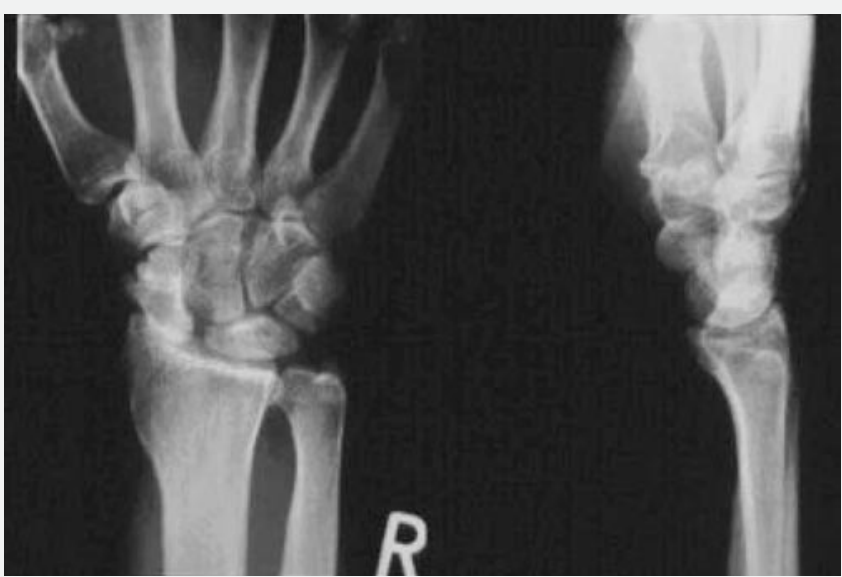

Abb. $14 \Delta$ Fortgeschrittene Handgelenkarthrose in Folge einer Skaphoidpseudarthrose (SNAC-wrist)
Abb. $13<C T$ des Skaphoids mit Humpback-Deformität (Abkippung) bei dislozierter Skaphoidfraktur en Carpus. Im Röntgenbild ist eine Panarthrose erkennbar.

Posttraumatische Rhizarthrose. Sie tritt z. B. nach Fraktur der Basis des 1. Mittelhandknochens auf und geht mit Krepitation und Druckschmerz über dem Daumensattelgelenk einher.

Posttraumatische STT-Arthrose. Es handelt sich um eine Arthrose zwischen
Skaphoid, Trapezium und Trapezoideum, für die ein Druckschmerz über dem STTGelenk typisch ist.

Arthrose des Os pisiforme. Es kommt zu Schmerzen beim Druck auf das Os pisiforme mit Schiebeschmerz des Os pisiforme.

Posttraumatisches Karpaltunnelsyndrom. Es kommt zu Sensibilitätsstörun- gen von Daumen, Zeige- und Mittelfinger, z. B. bei distaler Radiusfraktur oder perilunärer Luxation.

\section{Posttraumatisches Loge-de-Gyon-} Syndrom. Es äußert sich in einer Krallenstellung von Ring- und Kleinfinger, meist ohne sensible Störungen, z. B. bei Fraktur des Hamulus ossis hamati oder Hypothenar-Hammer-Syndrom. 


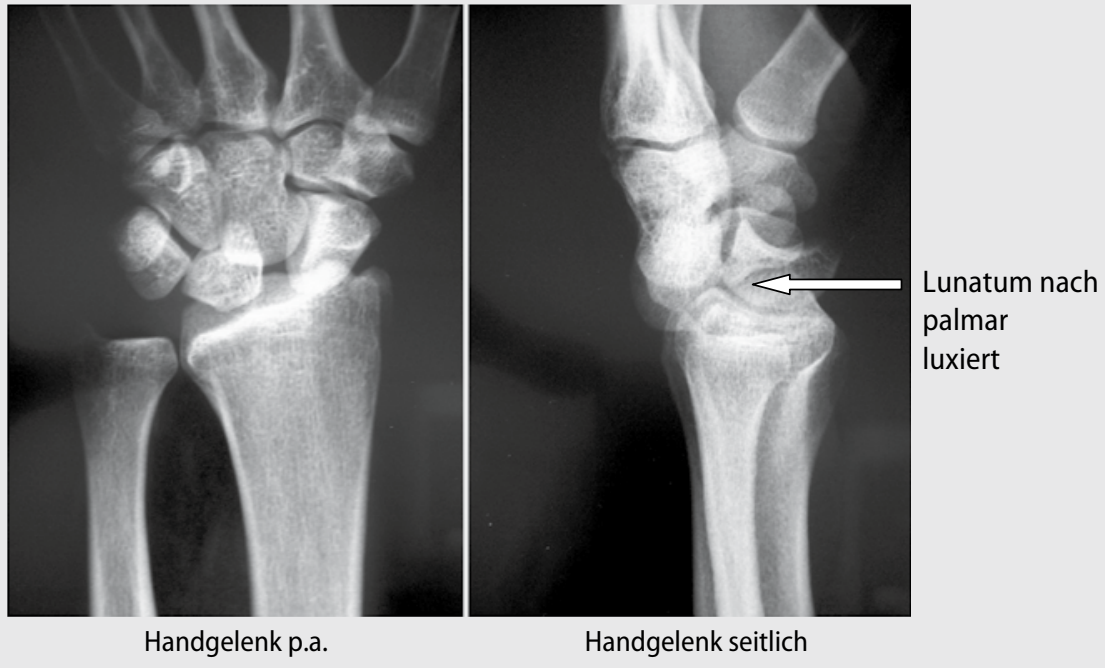

Abb. $15 \Delta$ Transstyloidale perilunäre Luxationsfraktur

Symptomatische mediokarpale Instabilität. Sie geht mit Bewegungsschmerzen im Handgelenk nach Sturz bei laxem Bandapparat einher.

\section{Extraskaphoidale Handwurzelfraktu-}

ren. Typisch sind persistierende Schmerzen der Handwurzel bei oft nicht aussagekräftigem Röntgenbild. Zur Abklärung wird ein CT empfohlen [2].

Perilunäre Luxationsfraktur. Ursache ist ein heftiges Aufprall- oder Anpralltrauma, Symptome sind eine Schwellung und Schmerzen im gesamten Carpus.

In der p.-a.-Röntgenaufnahme zeigen sich ein dreieckig konfiguriertes Lunatum und eine Fraktur des Processus styloideus radii und/oder des Skaphoids, im seitlichen Röntgenbild ist das Lunatum nach palmar luxiert (• Abb. 15) [2]. Es ist eine sofortige Reposition erforderlich.

Essex-Lopresti-Verletzung. Es handelt sich um eine komplexe Verletzung des Unterarms mit Radiusköpfchenfraktur und Zerreißung der Membrana interossea sowie Sprengung des DRUG [2]. Sie muss bei klinischem Verdacht immer ausgeschlossen werden, um gravierende Folgeschäden zu vermeiden.

\section{Fazit}

Sowohl die Kenntnis der Anatomie, der Biomechanik und der klinischen Untersuchungstechniken des Handgelenks als auch die Fortschritte in der bildge- benden Diagnostik, insbesondere der Arthroskopie, sind wichtige Voraussetzungen zur Interpretation und korrekten klinischen Beurteilung des schmerzhaften Handgelenks.

\section{Korrespondenzadresse}

\section{Prof. Dr. G. Germann}

Klinik für Hand, Plastische und Rekonstruktive Chirurgie - Schwerbrandverletztenzentrum, BG-Unfallklinik Ludwigshafen,

Klinik für Plastische und Handchirurgie an der Universität Heidelberg,

Ludwig-Guttmann-Straße 13,

67071 Ludwigshafen

guenter.germann@urz.uni-heidelberg.de

Interessenkonflikt. Der korrespondierende Autor gibt an, dass kein Interessenkonflikt besteht.

\section{Literatur}

1. Bickert B, Sauerbier M, Germann G (1997) Die klinische Untersuchung des verletzten Handgelenkes. Zentralbl Chir 122: 1010-1015

2. Green DP (2005) Green's operative hand surgery, 5th edn. Elsevier Churchill Livingstone, Philadelphia

3. Karle M, Mayer B, Kitzinger HB et al. (2005) Scaphoidfrakturen - operative oder konservative Behandlung? Eine CT-kontrollierte Klassifikation. Handchir Mikrochir Plast Chir 37: 260-266

4. King GJW, Mc Murtry Y (1996) Physical examination of the wrist and hand. In: Gilula LA, Yin Y (eds) Imaging of the wrist and hand. Saunders, Philadelphia

5. Krimmer H (1998) Rund um den Ellenkopf - UInokarpales Gelenkkompartiment und distales Radioulnargelenk als therapeutische Problemzone. Handchir Mikrochir Plast Chir 30: 345

6. La Stayo P,Howell J (1995) Clinical provocative tests in evaluation wrist pain: a descriptive study. J Hand Ther 8: 10-17
7. Larsen CF, Amadio PC, Gilula LA et al. (1995) Analysis of carpal instability: 1. description of the sceme. J Hand Surg [Am] 20A: 757-764

8. Meier R, Schmitt R, Krimmer H (2005) Wrist lesions in MRI arthrography compared with wrist arthroscopy. Handchir Mikrochir Plast Chir 37: 85-89

9. Müller M, Germann G, Sauerbier M (2004) Aktuelle Therapiekonzepte bei Arthrose im distalen Radioulnargelenk. Chir Allgemeine 5: 211-216

10. Nichols CM, Cheng Ch (2006) Update on the evaluation of wrist pain. Scientific 103: 293-296

11. Palmer AK, Werner FW (1981) The triangular fibrocartilage complex of the wrist - Anatomy and function. J Hand Surg [Am] 6: 153-162

12. Sauerbier M, Müller M (2007) Skaphoidfrakturen: Diagnostik, Zugangswege, Komplikationen. Zentralbl Chir 132: W42-W53

13. Sauerbier M, Tränkle M, Linser G et al. (2000) Midcarpal arthrodesis with complete scaphoid excision and interposition bone graft in the treatment of advanced carpal collapse (SNAC/SLAC-wrist): operative technique and outcome assessment. J Hand Surg [Br] 25: 341-345

14. Schmidt HM, Lanz U (1992) Chirurgische Anatomie der Hand. Hippokrates, Stuttgart

15. Schmidt HM (1998) Chirurgische Anatomie des distalen Radioulnargelenkes und des ulnokarpalen Gelenkkompartimentes. Handchir Mikrochir Plast Chir 30: 346-350

16. Tay SC, Tomita K, Berger RA (2007) The „ulnar fovea sign" for defining ulnar wrist pain: an analysis of sensitivity and specificity. J Hand Surg [Am] 32: 438-444

17. Watson HK, Ashmead D, Makhlouf MV (1988) Examination of the scaphoid. J Hand Surg [Am] 13A: 657-660

18. Zanetti M, Saupe N, Nagy L (2007) Role of MR imaging in chronic wrist pain. Eur Radiol 17: 927-938 